Article

\title{
The Novel [4,5-e][1,3]Diazepine-4,8-dione and Acyclic Carbamoyl Imino-Ureido Derivatives of Imidazole: Synthesis, Anti-Viral and Anti-Tumor Activity Evaluations
}

\author{
Karlo Wittine ${ }^{1}$, Kristina Poljak ${ }^{1}$, Matea Kovač ${ }^{1}$, Damjan Makuc ${ }^{2,3}$, Janez Plavec ${ }^{2,3}$, \\ Jan Balzarini ${ }^{4}$, Tamara Martinović ${ }^{5}$, Sandra Kraljević Pavelić ${ }^{5}$, Krešimir Pavelić ${ }^{5}$ \\ and Mladen Mintas ${ }^{1, *}$
}

1 Department of Organic Chemistry, Faculty of Chemical Engineering and Technology,

University of Zagreb, Marulićev trg 20, Zagreb HR-10000, Croatia;

E-Mails: kwittine@fkit.hr (K.W.); polly3112@net.hr (K.P.); matea.kovac@hotmail.com (M.K.)

2 Slovenian NMR Centre, National Institute of Chemistry, Hajdrihova 19, P. O. B. 660,

Ljubljana SI-1001, Slovenia; E-Mails: damjan.makuc@ki.si (D.M.); janez.plavec@ki.si (J.P.)

3 EN-FIST Centre of Excellence, Dunajska 156, Ljubljana SI-1001, Slovenia

4 Rega Institute for Medical Research, KU Leuven, Minderbroedersstraat 10,

Leuven B-3000, Belgium; E-Mail: jan.balzarini@rega.kuleuven.be (J.B.)

5 Department of Biotechnology, University of Rijeka, Radmile Matejčić 2, Rijeka 51000, Croatia;

E-Mails: tamara.martinovic@uniri.hr (T.M.); sandrakp@biotech.uniri.hr (S.K.);

pavelic@biotech.uniri.hr (K.P.)

* Author to whom correspondence should be addressed; E-Mail: mmintas@fkit.hr;

Tel.: +385-1-4597-214; Fax: +385-1-4597-250.

Received: 29 August 2013; in revised form: 5 October 2013 / Accepted: 17 October 2013 /

Published: 30 October 2013

\begin{abstract}
In the present paper, we report on the synthesis, and in vitro antiviral and cytostatic activities of a series of novel imidazole[4,5-e][1,3]diazepine-4,8-dione (compounds 9-11) and acyclic carbamoyl imino-ureido imidazole (compounds 12 and 13) derivatives. These new type of chemical entities showed no significant activity on the broad spectrum of DNA and RNA viruses. Results of antiproliferative assays performed on a panel of selected human tumor cell lines revealed that only compounds $\mathbf{1}$ and $\mathbf{5}$ showed moderate and selective cytostatic effect against HeLa cells $\left(\mathrm{IC}_{50}=24\right.$ and $\left.32 \mu \mathrm{M}\right)$ with no concomitant cytotoxic effects on human normal fibroblasts (BJ). Importantly, an imidazole derivative containing a pyrrolidine moiety linked via an ethylenic spacer (3) showed a selective cytostatic effect toward cervical carcinoma (HeLa) cells $\left(\mathrm{IC}_{50}=9.5 \mu \mathrm{M}\right)$ with
\end{abstract}


no apparent cytotoxicity on human normal fibroblasts (BJ). This compound can be therefore considered as a potential anti-tumor lead compound for further synthetic structure optimization.

Keywords: imidazole; [4,5-e][1,3]diazepine; anti-RSV; anti-tumor

\section{Introduction}

It has been found that infection with respiratory syncytial virus (RSV), which manifests primarily as bronchiolitis or viral pneumonia, is the leading cause of lower respiratory tract infections (LRTIs) in infants and young children [1]. Ribavirin still is the only antiviral agent approved for the treatment of RSV infection, but due to efficacy and toxicity issues, it has only limited utility [2]. There is a clear need for new anti-RSV therapeutics, with improved efficacy and safety for broader applications [3]. Powell and his colleagues have recently identified a new class of RSV inhibitors, namely 1,4-benzodiazepines [4], which eventually led to identification of RSV604 as a clinical candidate [5].

Furthermore, imidazole is an entity incorporated into many important biological molecules with a wide range of pharmacological activity. In the field of drug discovery the imidazole scaffold is widely used in the drug design strategy and imidazoles are generally well known as anticancer agents as well [6].

Moreover, heterocycles containing an imidazo[4,5-e][1,3]diazepine ring system have already shown potent in vitro activity at low micromolar concentrations against lung, breast, ovarian and prostate cancer cell lines [7]. Also, in vitro inhibitory activity of a number of ring-expanded nucleosides against NTPase/helicases of a family of Flaviviridae have been reported. Compounds containing an imidazo[4,5-e][1,3]diazepine-4,8-dione ring system have exhibited potent activities against HBV and HCV [8]. Ring-expanded nucleosides employing a fused imidazo[4,5-e][1,3]diazepine ring system show promising anti-measles virus activity at submicromolar or micromolar concentration levels with no apparent toxicity to the host cell line [9].

In the light of these findings we efficiently synthesized a series of new $1 H$-imidazole-4,5-dicarboxylic acid/amide derivatives (1-8), imidazo[4,5-e][1,3]diazepine-4,8-dione derivatives (9-11) and carbamoyl imino-ureido derivatives of imidazole (12 and 13) (Figure 1) and evaluated their antiviral and cytostatic activity potency.

\section{Results and Discussion}

\subsection{Chemistry}

The synthesis of new $1 H$-imidazole-4,5-dimethyl dicarboxylate (1-4), $1 H$-imidazole-4,5-diamide (5-8), imidazole[4,5-e][1,3]diazepine-4,8-dione (9-11) and acyclic carbamoyl imino-ureido imidazole derivatives (12 and $\mathbf{1 3})$ is performed according to the Figure 2. 
Figure 1. New $1 H$-imidazole-4,5-dicarboxylic acid/amide derivatives (1-8), imidazo[4,5-e][1,3]diazepine-4,8-dione derivatives (9-11) and carbamoyl imino-ureido derivatives of imidazole (12 and 13).
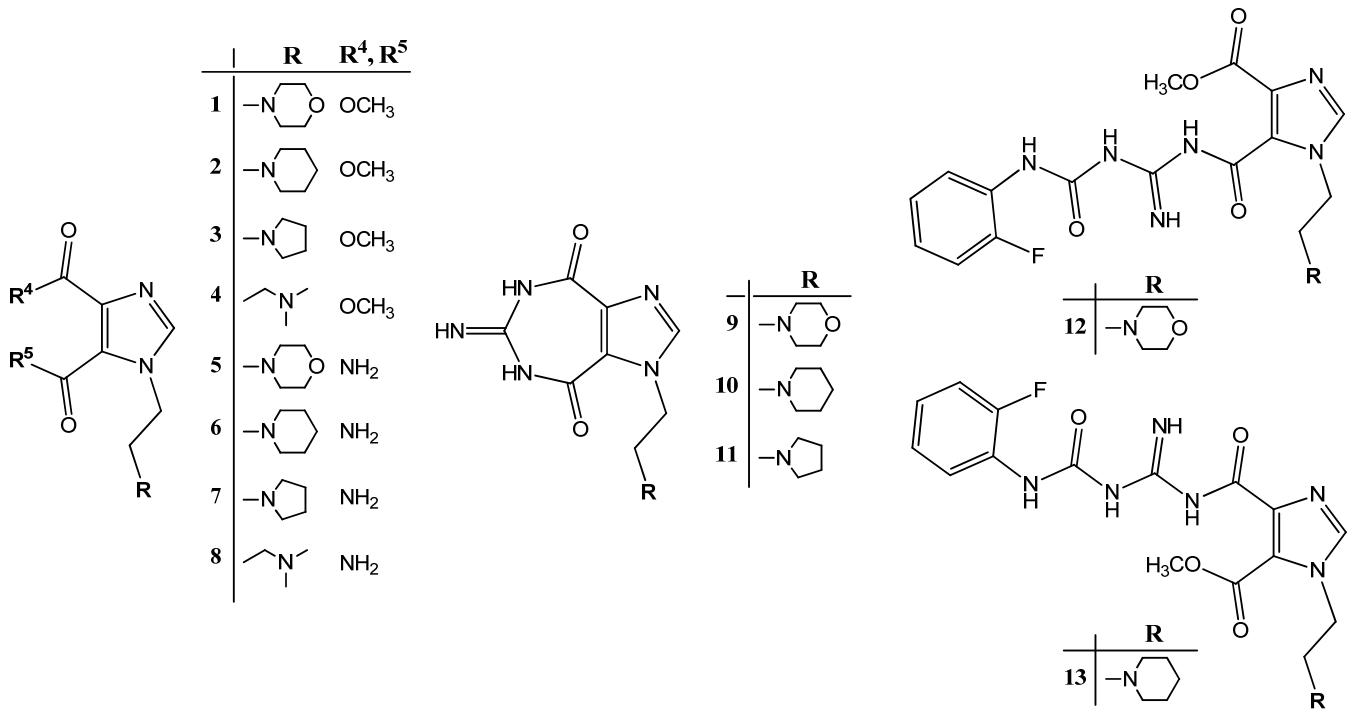

Figure 2. Synthesis of imidazo[4,5-e][1,3]diazepine-4,8-dione (9-11) and ureido-imino-carbamoyl-imidazole derivatives (12-13). Reagents and conditions: (i) $\mathrm{K}_{2} \mathrm{CO}_{3}, \mathrm{CH}_{3} \mathrm{CN}$, r.t.; (ii) $\mathrm{NH}_{3}, \mathrm{MeOH}$, r.t.; (iii) guanidine hydrochloride, $\mathrm{NaOMe}$, $\mathrm{MeOH}$, r.t.; (iv) 2-fluorophenyl isocyanate, DMF, r.t.

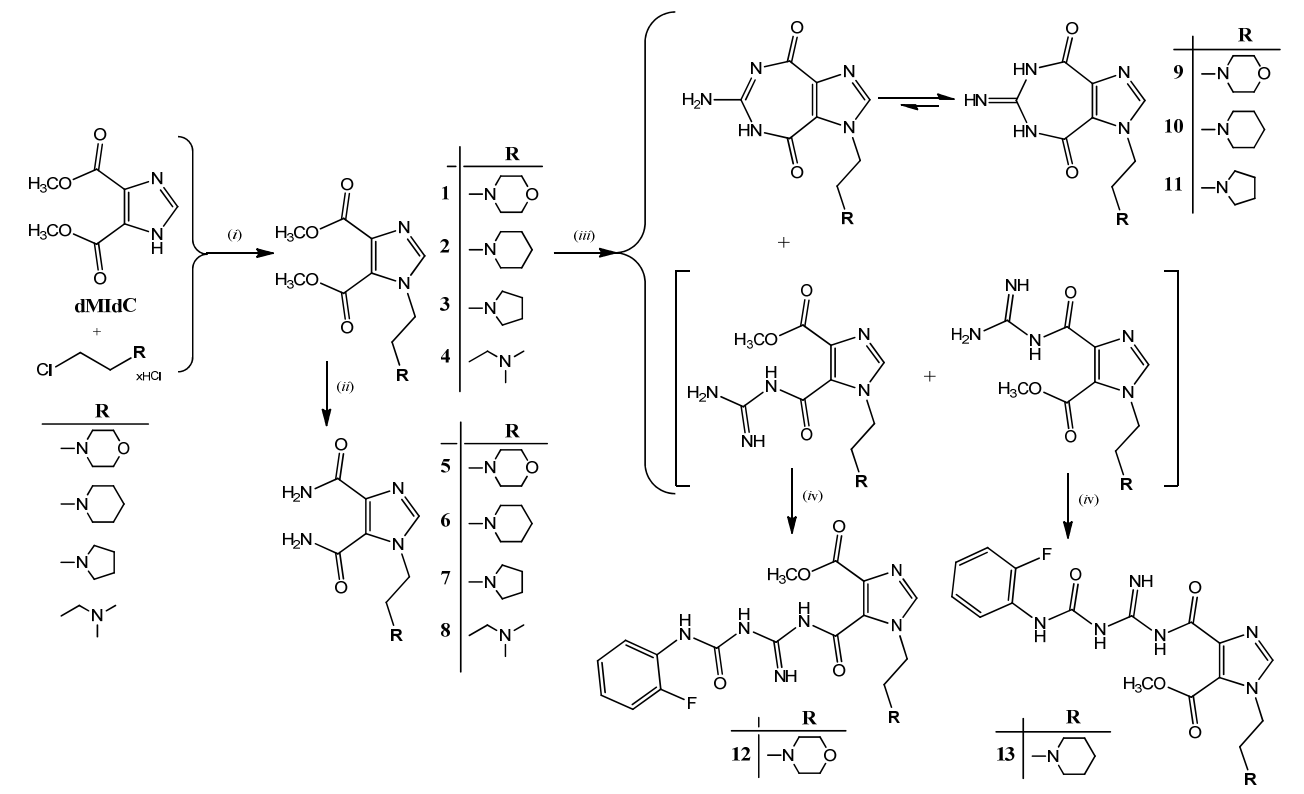

Reaction of 4,5-dimethyl $1 \mathrm{H}$-imidazole-dicarboxylate (dMIdC) with $\mathrm{K}_{2} \mathrm{CO}_{3}$ and different 2-chloroalkylamine hydrochlorides, that is 4-(2-chloroethyl)morpholine, 4-(2-chloroethyl)piperidine, 4-(2-chloroethyl)pyrrolidine and 3-dimethylaminopropyl chloride gave 4,5-dimethyl dicarboxylate derivatives (1-4) with alkylamino chains at the $\mathrm{N}-1$ of the imidazole ring. $1 \mathrm{H}$-imidazo-4,5-diamide derivatives (5-7) were obtained by ammonolysis of diesters 1-4 in polar protic solvent. 
Time-controlled reaction of compounds 1-3 with guanidine-hydrochloride and sodium-methoxide gave cyclized imidazo[4,5-e]diazepine-4,8-dione derivatives (9-11) and a mixture of two regioisomeric guanidino carbamoyl-imidazole derivatives which in subsequent in situ reaction with 2-fluorophenyl isocyanate in DMF afforded carbamoyl imino-ureido derivatives 12 and 13. It is worth noting that the reaction of imidazo[4,5-e][1,3] diazepine-4,8-dione derivatives (9-11) with 2-fluorophenyl isocyanate did not gave the desired ureido-imidazo[4,5-e][1,3]diazepine-4,8-dione derivative. The main reason obviously lies in the fact that 6-amino-diazepine-4,8-dione molecules (9-11) are predominantly in solution in their imino tautomeric form as confirmed by NMR spectroscopy, which is inactive in the final step of the synthesis to create the ureido derivative of imidazo[4,5-e][1,3]diazepine-4,8-dione. Morpholine derivative $\mathbf{1 2}$ for which we believed to have a diazepine-4,8-dione structure and whose NMR spectrum indicated next to all diagnostic signals for the assumed structure the presence of an unidentified signal at approximately $3.8 \mathrm{ppm}$ attributable to a OMe group, which is consistent with the results of the determination of the molecular ion mass by high resolution mass spectrometry.

\subsection{Structural Properties}

The structures of 1-13 have been confirmed by ${ }^{1} \mathrm{H}$ and ${ }^{13} \mathrm{C}-\mathrm{NMR}$ spectra (Experimental). The analysis of the spectra was achieved on the basis of the chemical shift, signal multiplicity and integral values. ${ }^{19} \mathrm{~F}$ NMR resonances of $\mathbf{1 2}$ and $\mathbf{1 3}$ were well resolved (Experimental). ${ }^{1} \mathrm{H}$ decoupled ${ }^{13} \mathrm{C}$-NMR showed C-F coupling constants that enabled straightforward identification of fluorinated carbon atoms and their neighbors. Two sets of signals were observed for 9 in the ratio 4:1 as was estimated from the integral values of ${ }^{1} \mathrm{H}-\mathrm{NMR}$ signals (e.g., $\mathrm{CH}-5$ proton at $\delta 8.05$ and $7.69 \mathrm{ppm}$ ). Two species were most probably detected due to presence of dynamic equilibrium between amine and imine tautomers $\left(\mathrm{C} 8-\mathrm{NH}_{2}\right.$ and $\left.\mathrm{C} 8=\mathrm{NH}\right) . \mathrm{C} 8$ chemical shifts of the major species showed value of $149.99 \mathrm{ppm}$, which is characteristic for the imine form. Therefore, imine form of $\mathbf{9}$ is the predominant species in solution. Likewise, broad ${ }^{13} \mathrm{C}$-NMR signals suggested presence of tautomer forms for $\mathbf{1 0}$ and $\mathbf{1 1}$. Imine forms are favored according to C8 chemical shift ( $\delta 149.70$ and $150.12 \mathrm{ppm}$ for $\mathbf{1 0}$ and 11, respectively).

The correlation signals observed in ${ }^{1} \mathrm{H}_{-}{ }^{13} \mathrm{C}$ HSQC and $\mathrm{HMBC}$ spectra allowed assignment of $\mathrm{C} 2$, $\mathrm{C} 3$ and C5 atoms. Interestingly, major chemical shifts differences between $\mathbf{1 2}$ and $\mathbf{1 3}$ were observed for $\mathrm{C} 2$ and $\mathrm{C} 3$ atoms. The correlation signals between methylene protons $\mathrm{CH}_{2}-1^{\prime}$ and $\mathrm{C} 2$ and $\mathrm{C} 5$ in combination with chemical shift values of imidazole carbon atoms suggested that $\mathrm{COOCH}_{3}$ group is attached to $\mathrm{C} 2(\delta 121.43 \mathrm{ppm})$ and fluoro-phenyl-ureido-imino-methyl-carbamoyl moiety to $\mathrm{C} 3$ $(\delta 145.53)$ in 12. On the other hand, $\mathrm{COOCH}_{3}$ in attached to $\mathrm{C} 3 \mathrm{in} \mathbf{1 3}$, which is indicated by $\mathrm{C} 2$ and $\mathrm{C} 3$ chemical shift ( $\delta 127.71$ and $134.19 \mathrm{ppm})$.

These spectroscopic results indicate that compounds 12 and 13 exist as regioisomers with respect to substitution at positions C-2 and C-3 of the imidazole moiety. This implies that both regioisomers are formed as indicated in Figure 2 and their subsequent in situ reaction gave compounds 12 and 13. Structural differences between $\mathbf{1 2}$ and 13, which were suggested by distinct NMR chemical shifts of C2 and C3, were assessed by NOESY experiments. Unfortunately, only trivial NOESY cross-peaks were observed for 12 and $\mathbf{1 3}$. $\mathrm{COOCH}_{3}$ group showed no NOESY signals with methylene protons and therefore no particular conformational preferences could be established for these compounds. 


\subsection{Biological Results}

\subsubsection{Antiviral Activity}

Compounds 1-3, 5-7 and 9-13 were evaluated for their antiviral activity against a wide variety of DNA and RNA viruses, including herpes simplex virus type 1 (HSV-1) (KOS), HSV-2 (G), vaccinia virus and vesicular stomatitis virus in HEL cells, parainfluenza-3, reovirus-1, Sindbis, Coxsackie B4, and Punta Toro virus in Vero cells, vesicular stomatitis virus, Coxsackie virus B4, and respiratory syncytial virus in HeLa cells and influenza A (H1N1; H3N2) and influenza B viruses in MDCK cells. Unfortunately, none of the compounds showed pronounced antiviral activity at subtoxic concentrations. No cytotoxicity for all evaluated compounds on HEL, Vero, HeLa and MDCK cell cultures was observed (data not shown).

\subsubsection{Cytostatic Activity}

Compounds 1-12 were evaluated for their antiproliferative effect against several malignant tumor cell lines: cervical carcinoma (HeLa), colorectal adenocarcinoma, metastatic (SW 620), breast epithelial adenocarcinoma, metastatic (MCF-7) and human hepatocarcinoma (HepG2) cells and compared with their effects on the growth of normal human skin fibroblasts (BJ) (Table 1). The imidazole[4,5-e][1,3]diazepine-4,8-dione derivative linked with a pyrrolidine ligand (11) showed a modest cytostatic effect on colon cancer cells (SW620) $\left(\mathrm{IC}_{50}=20 \mu \mathrm{M}\right)$ while imidazole derivative containing a pyrrolidine moiety linked via an ethylenic spacer (3) showed specific antiproliferative effect on HeLa cells $\left(\mathrm{IC}_{50}=9.5 \mu \mathrm{M}\right)$. However, both compounds 11 and $\mathbf{3}$ exerted moderate growth inhibition only at the highest tested concentrations $(10$ and $100 \mu \mathrm{M})$. Other compounds exerted weak or no antproliferative effects on tested cell lines.

Table 1. Inhibitory effects of compounds 1-12 on the growth of malignant tumor cell lines in comparison with their effect on normal human skin fibroblasts (BJ).

\begin{tabular}{|c|c|c|c|c|c|c|}
\hline \multicolumn{7}{|c|}{ Tumor cell growth $\mathrm{IC}_{50}{ }^{\mathrm{a}}(\mu \mathrm{M})$} \\
\hline \multirow{2}{*}{ Compd. } & \multirow{2}{*}{ Structure } & \multicolumn{5}{|c|}{$\underline{\text { Cell lines }}$} \\
\hline & & MCF-7 & HepG2 & SW620 & HeLa & BJ \\
\hline 1 & & $>100$ & $>100$ & $>100$ & 24 & $>100$ \\
\hline 2 & & $>100$ & $>100$ & $>100$ & $>100$ & $>100$ \\
\hline 3 & & $>100$ & $>100$ & $>100$ & 9.5 & $>100$ \\
\hline
\end{tabular}


Table 1. Cont.

\begin{tabular}{|c|c|c|c|c|c|c|}
\hline \multicolumn{7}{|c|}{ Tumor cell growth $\mathrm{IC}_{50}{ }^{\mathrm{a}}(\boldsymbol{\mu M})$} \\
\hline \multirow{2}{*}{ Compd. } & \multirow{2}{*}{ Structure } & \multicolumn{5}{|c|}{ Cell lines } \\
\hline & & MCF-7 & HepG2 & SW620 & HeLa & BJ \\
\hline 4 & & $>100$ & $>100$ & $>100$ & $>100$ & $>100$ \\
\hline 5 & & $>100$ & $>100$ & $>100$ & 32 & $>100$ \\
\hline 6 & & $>100$ & $>100$ & $>100$ & $>100$ & $>100$ \\
\hline 7 & & $>100$ & $>100$ & $>100$ & $>100$ & $>100$ \\
\hline 8 & & $>100$ & $>100$ & $>100$ & $>100$ & $>100$ \\
\hline 9 & & $>100$ & $>100$ & $>100$ & $>100$ & $>100$ \\
\hline 10 & & $>100$ & 41 & 31 & 31 & $>100$ \\
\hline 11 & & $>100$ & $>100$ & 20 & $>100$ & $>100$ \\
\hline 12 & & $>100$ & $>100$ & $>100$ & $>100$ & $>100$ \\
\hline
\end{tabular}

${ }^{\mathrm{a}} \mathrm{IC}_{50} ; 50 \%$ inhibitory concentration, or compound concentration required to inhibit tumor cell proliferation by $50 \%$. 


\section{Experimental}

\subsection{General Materials and Methods}

All commercially available chemicals were purchased from Sigma Aldrich (Hamburg, Germany) and used without purification. All solvents were analytical grade purity and dried. Methanol $\left(\mathrm{CH}_{3} \mathrm{OH}\right)$ was stored over $3 \AA$ molecular sieves. Dimethylformamide (DMF) was stored over $4 \AA$ molecular sieves. Dichloromethane $\left(\mathrm{CH}_{2} \mathrm{Cl}_{2}\right)$ was refluxed over phosphorus pentoxide $\left(\mathrm{P}_{2} \mathrm{O}_{5}\right)$, distilled and stored over $4 \AA$ molecular sieves. Merck silica gel $60 \mathrm{~F}_{254}$ plates were used for thin-layer chromatography. Column chromatography was performed with Merck silica gel $(0.063-0.200 \mathrm{~mm})$, with dichloromethane/methanol as eluent. ${ }^{1} \mathrm{H}$ and ${ }^{13} \mathrm{C}-\mathrm{NMR}$ spectra were recorded on a Varian Gemini 300 spectrometer (Institute Ruđer Bošković, Zagreb, Croatia) and Varian NMR System 600 and Varian Unity Inova 300 and Agilent Technologies DD2 $300 \mathrm{MHz}$ NMR spectrometers (National Institute of Chemistry, Ljubljana, Slovenia) Samples were measured in $\mathrm{CDCl}_{3}$ and DMSO- $d_{6}$ solutions at $25^{\circ} \mathrm{C}$ in $5 \mathrm{~mm}$ NMR tubes. Chemical shifts $(\delta)$ in ppm were referred to TMS. High performance LC was performed on Agilent 1100 series system with UV detection (photodiode array detector) using Zorbax C18 reverse-phase analytical column $(2.1 \times 30 \mathrm{~mm} ; 3.5 \mu \mathrm{m})$. All compound used for biological evaluation showed $>95 \%$ purity in this HPLC system. The electron impact mass spectra and the purity of compounds were assessed by using Agilent Technologies 6410 Triple Quad LC/MS instrument equipped with electrospray interface and triple quadrupole analyzer (LC-MS/MS).

\subsection{Procedures for the Preparations of Compounds}

\subsubsection{General Procedure for Synthesis of $\mathbf{1}-\mathbf{4}$}

To a solution of 4,5-dimethyl $1 \mathrm{H}$-imidazole-dicarboxylate $(1 \mathrm{~g} ; 5.43 \mathrm{mmol})$ and $\mathrm{K}_{2} \mathrm{CO}_{3}(1.5 \mathrm{~g}$; $10.86 \mathrm{mmol}$ ) in $\mathrm{CH}_{3} \mathrm{CN}(30 \mathrm{~mL})$ is added 4-(2-chloroethyl)morpholine hydrochloride (for cmpd 1), 4-(2-chloroethyl)piperidine hydrochloride (for cmpd 2), 4-(2-chloroethyl)pyrrolidine hydrochloride (for cmpd 3) or 3-dimethylaminopropyl chloride hydrochloride (for cmpd 4) (5.43 mmol). Reaction mixture is stirred at room temperature for $48 \mathrm{~h}$. The solvent is evaporated and the crude product simply extracted with $\mathrm{CH}_{2} \mathrm{Cl}_{2}$ or purified by silica gel column chromatography $\left(\mathrm{CH}_{2} \mathrm{Cl}_{2} / \mathrm{CH}_{3} \mathrm{OH}=20 / 1\right)$.

1-(2-Morpholin-4-yl-ethyl)-1H-imidazole-4,5-dicarboxylic acid dimethyl ester (1): Following general procedure compound 1 is obtained as a yellow oil (1.68 g; 52.23\%); MS $m / z: 297.1(\mathrm{M}+1)$. ${ }^{1} \mathrm{H}-\mathrm{NMR}$ $\left(\mathrm{DMSO}_{-} d_{6}\right): \delta 2.37\left(4 \mathrm{H}, \mathrm{m}, \mathrm{CH}_{2}-4^{\prime}\right), 2.58\left(2 \mathrm{H}, \mathrm{t}, J=5.8, \mathrm{CH}_{2}-2^{\prime}\right), 3.52\left(4 \mathrm{H}, \mathrm{m}, \mathrm{CH}_{2}-5^{\prime}\right), 3.79(3 \mathrm{H}, \mathrm{s}$, $\left.\left.\mathrm{COOCH}_{3}-3\right), 3.84\left(3 \mathrm{H}, \mathrm{s}, \mathrm{COOCH}_{3}-2\right), 4.29\left(2 \mathrm{H}, \mathrm{t}, J=5.7, \mathrm{CH}_{2}-1\right)^{\prime}\right), 7.97 \mathrm{ppm}(1 \mathrm{H}, \mathrm{s}, \mathrm{CH}-5)$. ${ }^{13} \mathrm{C}-\mathrm{NMR}\left(\mathrm{DMSO}-d_{6}\right): \delta 43.63\left(\mathrm{C} 1\right.$ '), $52.29\left(\mathrm{COOCH}_{3}-3\right), 52.86\left(\mathrm{COOC} \mathrm{H}_{3}-2\right), 53.63(\mathrm{C} 4), 58.72$ (C2'), 66.57 (C5'), 124.99 (C2), 136.24 (C3), 141.58 (C5), $160.78\left(\underline{\mathrm{COOCH}}_{3}\right), 163.44$ ppm $\left(\mathrm{COOCH}_{3}\right)$.

1-(2-Piperidin-1-yl-ethyl)-1H-imidazole-4,5-dicarboxylic acid dimethyl ester (2): Following general procedure compound 2 is obtained as a pale yellow oil (1.32 g; 83.36\%); MS m/z: $296.2(\mathrm{M}+1)$. ${ }^{1} \mathrm{H}-\mathrm{NMR}\left(\mathrm{DMSO}-d_{6}\right): \delta 1.25-1.45\left(6 \mathrm{H}, \mathrm{m}, 2 \times \mathrm{CH}_{2}-5^{\prime}\right.$ and $\left.\mathrm{CH}_{2}-6^{\prime}\right), 2.30\left(4 \mathrm{H}, \mathrm{m}, \mathrm{CH}_{2}-4^{\prime}\right), 2.51(2 \mathrm{H}, \mathrm{t}$, $\left.J=5.7, \mathrm{CH}_{2}-2^{\prime}\right), 3.77\left(3 \mathrm{H}, \mathrm{s}, \mathrm{COOCH}_{3}-3\right), 3.83\left(3 \mathrm{H}, \mathrm{s}, \mathrm{COOCH}_{3}-2\right), 4.25\left(2 \mathrm{H}, \mathrm{t}, J=5.9, \mathrm{CH}_{2}-1^{\prime}\right)$,

7.92 ppm (1H, s, CH-5). ${ }^{13} \mathrm{C}-\mathrm{NMR}$ (DMSO- $\left.d_{6}\right): \delta 24.29$ (C6'), 25.94 (C5'), 44.09 (C1'), 
$52.28\left(\mathrm{COOC}_{3}-3\right), 52.84\left(\mathrm{COO}_{3}{ }_{3}-2\right), 54.47$ (C4'), 59.10 (C2'), 125.17 (C2), 136.05 (C3), $141.47(\mathrm{C} 5), 160.79\left(\underline{\mathrm{COOCH}_{3}}\right), 163.42 \mathrm{ppm}\left(\underline{\mathrm{COOCH}}_{3}\right)$.

1-(2-Pyrrolidin-1-yl-ethyl)-1H-imidazole-4,5-dicarboxylic acid dimethyl ester (3): Following general procedure compound 3 is obtained as a pale yellow oil $(1.11 \mathrm{~g} ; 72.98 \%)$; MS m/z: $282.1(\mathrm{M}+1)$. ${ }^{1} \mathrm{H}-\mathrm{NMR}$ (DMSO- $\left.d_{6}\right): \delta 1.69\left(4 \mathrm{H}, \mathrm{m}, \mathrm{CH}_{2}-5^{\prime}\right), 2.43\left(4 \mathrm{H}, \mathrm{m}, \mathrm{CH}_{2}-4{ }^{\prime}\right), 2.70\left(2 \mathrm{H}, \mathrm{t}, J=6.1, \mathrm{CH}_{2}-2^{\prime}\right)$, $3.78\left(3 \mathrm{H}, \mathrm{s}, \mathrm{COOCH}_{3}-3\right), 3.83\left(3 \mathrm{H}, \mathrm{s}, \mathrm{COOCH}_{3}-2\right), 4.27\left(2 \mathrm{H}, \mathrm{t}, J=6.1, \mathrm{CH}_{2}-1^{\prime}\right), 7.96 \mathrm{ppm}(1 \mathrm{H}, \mathrm{s}$, $\mathrm{CH}-5) .{ }^{13} \mathrm{C}-\mathrm{NMR}\left(\mathrm{DMSO}-d_{6}\right): \delta 23.64\left(\mathrm{C} 5^{\prime}\right), 45.64\left(\mathrm{C} 1^{\prime}\right), 52.30\left(\mathrm{COOCH}_{3}-3\right), 52.87\left(\mathrm{COOCH}_{3}-2\right)$, 53.96 (C4'), 56.20 (C2'), 125.18 (C2), 136.06 (C3), 141.41 (C5), $160.78\left(\right.$ COOCH $\left._{3}\right), 163.39$ ppm $\left(\underline{\left.\mathrm{COOCH}_{3}\right)}\right.$.

1-(3-Dimethylamino-propyl)-1H-imidazole-4,5-dicarboxylic acid dimethyl ester (4): Following general procedure compound 4 is obtained as a pale yellow oil $(0.75 \mathrm{~g} ; 75.0 \%)$; MS m/z: $270.1(\mathrm{M}+1)$. ${ }^{1} \mathrm{H}-\mathrm{NMR}\left(\mathrm{DMSO}-d_{6}\right): \delta 1.84\left(2 \mathrm{H}, \mathrm{m}, \mathrm{CH}_{2}-2^{\prime}\right), 2.16\left(6 \mathrm{H}, \mathrm{s}, 2 \times \mathrm{CH}_{3}\right), 2.29\left(2 \mathrm{H}, \mathrm{t}, J=6.5, \mathrm{CH}_{2}-3^{\prime}\right), 3.76$ $\left(3 \mathrm{H}, \mathrm{s}, \mathrm{COOCH}_{3}-3\right), 3.81\left(3 \mathrm{H}, \mathrm{s}, \mathrm{COOCH}_{3}-2\right), 4.18\left(2 \mathrm{H}, \mathrm{t}, J=7.1, \mathrm{CH}_{2}-1^{\prime}\right), 7.96 \mathrm{ppm}(1 \mathrm{H}, \mathrm{s}, \mathrm{CH}-5)$. ${ }^{13} \mathrm{C}-\mathrm{NMR}\left(\mathrm{DMSO}-d_{6}\right): \delta 28.21\left(\mathrm{C}^{\prime}\right), 44.74\left(\mathrm{C} 1^{\prime}\right), 45.04\left(\mathrm{CH}_{3}\right) 52.34\left(\mathrm{COOCH}_{3}-3\right), 52.99\left(\mathrm{COOCH}_{3}-2\right)$, 55.73 (C3'), 124.89 (C2), 136.37 (C3), 141.29 (C5), $160.67\left(\underline{C O O C H}_{3}\right), 163.39$ ppm $\left(\underline{\mathrm{COOCH}_{3}}\right)$.

\subsubsection{General Procedure for Synthesis of 5-8}

To a stirred solution of compounds $\mathbf{1 - 4}(500 \mathrm{mg} ; 1.68 \mathrm{mmol})$ in anhydrous $\mathrm{CH}_{3} \mathrm{OH}(15 \mathrm{~mL})$ ammonia is introduced at $0{ }^{\circ} \mathrm{C}$. After saturation a reaction mixture is stirred overnight at room temperature. The solvent and excess ammonia are removed under reduced pressure and the crude product purified by silica gel column chromatography $\left(\mathrm{CH}_{2} \mathrm{Cl}_{2} / \mathrm{CH}_{3} \mathrm{OH}=2 / 1\right)$.

1-(2-Morpholin-4-yl-ethyl)-1H-imidazole-4,5-dicarboxylic acid diamide (5): According to the general procedure compound 5 is obtained as a white crystals $\left(262 \mathrm{mg} ; 58.17 \%\right.$; m.p. $\left.=196-197{ }^{\circ} \mathrm{C}\right)$; $\mathrm{MS} \mathrm{m} / \mathrm{z}$ : $267.1(\mathrm{M}+1) .{ }^{1} \mathrm{H}-\mathrm{NMR}\left(\mathrm{DMSO}-d_{6}\right): \delta 2.39\left(4 \mathrm{H}, \mathrm{m}, \mathrm{CH}_{2}-4^{\prime}\right), 2.59\left(2 \mathrm{H}, \mathrm{t}, J=6.1, \mathrm{CH}_{2}-2^{\prime}\right)$, $3.51\left(4 \mathrm{H}, \mathrm{m}, \mathrm{CH}_{2}-5^{\prime}\right), 4.53\left(2 \mathrm{H}, \mathrm{t}, J=6.1, \mathrm{CH}_{2}-1^{\prime}\right), 7.53\left(1 \mathrm{H}, \mathrm{s}, \mathrm{CONH}_{2}-2\right), 7.75\left(1 \mathrm{H}, \mathrm{s}, \mathrm{CONH}_{2}-3\right)$, $7.88(1 \mathrm{H}, \mathrm{s}, \mathrm{CH}-5), 7.95\left(1 \mathrm{H}, \mathrm{s}, \mathrm{CONH}_{2}-3\right), 10.65$ ppm $\left(1 \mathrm{H}, \mathrm{s}, \mathrm{CONH}_{2}-2\right) .{ }^{13} \mathrm{C}-\mathrm{NMR}\left(\mathrm{DMSO}-d_{6}\right)$ : $\delta 43.93$ (C1'), 53.25 (C4'), 58.51 (C2'), 66.18 (C5'), 126.16 (C2), 136.90 (C3), 140.53 (C5), 160.66 (CO-2), 165.80 ppm (CO-3).

1-(2-Piperidin-1-il-etil)-1H-imidazol-4,5-diamide (6): According to the general procedure compound 6 is obtained as a white crystals (194 mg; 53.93\%; m.p. $\left.=200-201{ }^{\circ} \mathrm{C}\right) ; \mathrm{MS} \mathrm{m} / z: 265.2(\mathrm{M}+1)$. ${ }^{1} \mathrm{H}-\mathrm{NMR}$ (DMSO- $\left.d_{6}\right): \delta 1.35\left(2 \mathrm{H}, \mathrm{m}, \mathrm{CH}_{2}-6^{\prime}\right), 1.43\left(4 \mathrm{H}, \mathrm{m}, \mathrm{CH}_{2}-5^{\prime}\right), 2.34\left(4 \mathrm{H}, \mathrm{m}, \mathrm{CH}_{2}-4^{\prime}\right), 2.54(2 \mathrm{H}, \mathrm{t}$, $\left.J=6.2, \mathrm{CH}_{2}-2^{\prime}\right), 4.51\left(2 \mathrm{H}, \mathrm{t}, J=6.2, \mathrm{CH}_{2}-1^{\prime}\right), 7.51\left(1 \mathrm{H}, \mathrm{s}, \mathrm{CONH}_{2}-2\right), 7.74\left(1 \mathrm{H}, \mathrm{s}, \mathrm{CONH}_{2}-3\right)$, $7.85(1 \mathrm{H}, \mathrm{s}, \mathrm{CH}-5), 7.94\left(1 \mathrm{H}, \mathrm{s}, \mathrm{CONH}_{2}-3\right), 10.64 \mathrm{ppm}\left(1 \mathrm{H}, \mathrm{s}, \mathrm{CONH}_{2}-2\right) .{ }^{13} \mathrm{C}-\mathrm{NMR}\left(\mathrm{DMSO}-d_{6}\right)$ : $\delta 24.41$ (C6'), 26.08 (C5'), 44.90 (C1'), 54.55 (C4'), 59.30 (C2'), 126.64 (C2), 135.36 (C3), 140.98 (C5), 161.14 (CO-2), $166.31 \mathrm{ppm}(\mathrm{CO}-3)$.

1-(2-Piperidin-1-yl-ethyl)-1H-imidazole-4,5-dicarboxylic acid diamide (7): According to the general procedure compound 7 is obtained as a white crystals $\left(182 \mathrm{mg} ; 45.50 \%\right.$; m.p. $\left.=205-210{ }^{\circ} \mathrm{C}\right)$; $\mathrm{MS} \mathrm{m} / \mathrm{z}$ : $252.2(\mathrm{M}+1) .{ }^{1} \mathrm{H}-\mathrm{NMR}\left(\mathrm{DMSO}-d_{6}\right): \delta 1.65\left(4 \mathrm{H}, \mathrm{m}, \mathrm{CH}_{2}-5^{\prime}\right), 2.45\left(4 \mathrm{H}, \mathrm{m}, \mathrm{CH}_{2}-4{ }^{\prime}\right), 2.72(2 \mathrm{H}, \mathrm{t}, J=5.6$, 
$\left.\mathrm{CH}_{2}-2^{\prime}\right), 4.52\left(2 \mathrm{H}, \mathrm{t}, J=6.1, \mathrm{CH}_{2}-1^{\prime}\right), 7.53\left(1 \mathrm{H}, \mathrm{s}, \mathrm{CONH}_{2}-2\right), 7.75\left(1 \mathrm{H}, \mathrm{s}, \mathrm{CONH}_{2}-3\right), 7.88(1 \mathrm{H}, \mathrm{s}$, $\mathrm{CH}-5), 7.94\left(1 \mathrm{H}, \mathrm{s}, \mathrm{CONH}_{2}-3\right), 10.65$ ppm $\left(1 \mathrm{H}, \mathrm{s}, \mathrm{CONH}_{2}-2\right) .{ }^{13} \mathrm{C}-\mathrm{NMR}$ (DMSO-d $\left.{ }_{6}\right): \delta 23.16\left(\mathrm{C}^{\prime}\right)$, 46.03 (C1'), 53.52 (C4'), 55.97 (C2'), 126.11 (C2), 134.98 (C3), 140.42 (C5), 160.61 (CO-2), 165.80 ppm (CO-3).

1-(3-Dimethylamino-propyl)-1H-imidazole-4,5-dicarboxylic acid diamide (8): According to the general procedure compound 8 is obtained as a white crystals $\left(144 \mathrm{mg} ; 48.0 \%\right.$; m.p. $\left.=205-210{ }^{\circ} \mathrm{C}\right)$; MS $m / z: 240.1(\mathrm{M}+1) .{ }^{1} \mathrm{H}-\mathrm{NMR}\left(\mathrm{DMSO}-d_{6}\right): \delta 1.83\left(2 \mathrm{H}, \mathrm{m}, \mathrm{CH}_{2}-2^{\prime}\right), 2.11\left(6 \mathrm{H}, \mathrm{s}, 2 \times \mathrm{CH}_{3}\right), 2.14(2 \mathrm{H}$, $\left.\mathrm{t}, J=6.9, \mathrm{CH}_{2}-3^{\prime}\right), 4.42\left(2 \mathrm{H}, \mathrm{t}, J=7.0, \mathrm{CH}_{2}-1^{\prime}\right), 7.57\left(1 \mathrm{H}, \mathrm{s}, \mathrm{CONH}_{2}-2\right), 7.83\left(1 \mathrm{H}, \mathrm{s}, \mathrm{CONH}_{2}-3\right), 7.89$ $(1 \mathrm{H}, \mathrm{s}, \mathrm{CH}-5), 7.98\left(1 \mathrm{H}, \mathrm{s}, \mathrm{CONH}_{2}-3\right), 10.66 \mathrm{ppm}\left(1 \mathrm{H}, \mathrm{s}, \mathrm{CONH}_{2}-2\right) .{ }^{13} \mathrm{C}-\mathrm{NMR}\left(\mathrm{DMSO}-d_{6}\right): \delta 29.09$ $(\mathrm{C} 2 '), 45.50\left(\mathrm{CH}_{3}\right), 45.93$ (C3'), $56.31\left(\mathrm{Cl}^{\prime}\right), 126.71(\mathrm{C} 2), 135.60$ (C3), 140.61 (C5), 161.02 (CO-2), $166.25 \mathrm{ppm}(\mathrm{CO}-3)$.

\subsubsection{General Procedure for Synthesis of $\mathbf{9 - 1 1}$}

To a solution of guanidine hydrochloride $(5.38 \mathrm{mmol})$ in anhydrous methanol $(10 \mathrm{~mL})$ cooled to $0{ }^{\circ} \mathrm{C}$ is added a solution of sodium methoxide $(25 \mathrm{wt} \% ; 13 \mathrm{mmol})$. The reaction mixture is stirred for $30 \mathrm{~min}$ at $0{ }^{\circ} \mathrm{C}$. The sodium salt formed is removed by filtration, and the filtrate thus obtained is added to a solution of compounds $\mathbf{1}-\mathbf{3}(1.35 \mathrm{mmol})$ in anhydrous methanol $(5 \mathrm{~mL})$. Reaction mixture is stirred for $72 \mathrm{~h}$ at room temperature. The solvent is evaporated and the crude product purified by silica gel column chromatography $\left(\mathrm{CH}_{2} \mathrm{Cl}_{2} / \mathrm{CH}_{3} \mathrm{OH}=2 / 1\right)$.

6-Imino-1-(2-morpholin-4-yl-ethyl)-6,7-dihydro-1H,5H-1,3,5,7-tetraaza-azulene-4,8-dione (9): (55 mg; 13.99\%; m.p. > $\left.300{ }^{\circ} \mathrm{C}\right)$; MS m/z: $292.1(\mathrm{M}+1) .{ }^{1} \mathrm{H}-\mathrm{NMR}\left(\mathrm{DMSO}-d_{6}\right): \delta 2.38\left(4 \mathrm{H}, \mathrm{m}, \mathrm{CH}_{2}-4^{\prime}\right), 2.58$ $\left(2 \mathrm{H}, \quad \mathrm{m}, \mathrm{CH}_{2}-2^{\prime}\right), \quad 3.51 \quad\left(4 \mathrm{H}, \mathrm{m}, \mathrm{CH}_{2}-5^{\prime}\right), 4.48 \quad\left(2 \mathrm{H}, \quad \mathrm{m}, \mathrm{CH}_{2}-1^{\prime}\right), \quad 6.64 \quad(1 \mathrm{H}, \quad \mathrm{b}, \mathrm{NH})$, 7.37 (1H, b, NH), 8.04 (1H, s, CH-5), 10.66 ppm (1H, b, NH). ${ }^{13} \mathrm{C}-\mathrm{NMR}\left(\mathrm{DMSO}_{6}\right)$ : $\delta 43.18\left(\mathrm{Cl}^{\prime}\right)$, 53.19 (C4'), 58.27 (C2'), 66.15 (C5'), 129.7 (C2), 135.2 (C3), 143.41 (C5), 149.99 (C8), 160.27 and $161.63 \mathrm{ppm}(\mathrm{C} 6$ and $\mathrm{C} 10)$.

6-Imino-1-(2-piperidin-1-yl-ethyl)-6,7-dihydro-1H,5H-1,3,5,7-tetraaza-azulene-4,8-dione (10): (22 mg; 2.23\%; m.p. > $300{ }^{\circ} \mathrm{C}$ ); $\mathrm{MS} \mathrm{m} / z: 290.2(\mathrm{M}+1) .{ }^{1} \mathrm{H}-\mathrm{NMR}\left(\mathrm{DMSO}-d_{6}\right): \delta 1.36\left(2 \mathrm{H}, \mathrm{m}, \mathrm{CH}_{2}-6^{\prime}\right), 1.43$ $\left(4 \mathrm{H}, \mathrm{m}, \mathrm{CH}_{2}-5^{\prime}\right), 2.35\left(4 \mathrm{H}, \mathrm{m}, \mathrm{CH}_{2}-4^{\prime}\right), 2.55\left(2 \mathrm{H}, \mathrm{t}, J=6.2, \mathrm{CH}_{2}-2^{\prime}\right), 4.46\left(2 \mathrm{H}, \mathrm{t}, J=6.2, \mathrm{CH}_{2}-1^{\prime}\right), 6.48$ $(1 \mathrm{H}, \mathrm{b}, \mathrm{NH}), 7.55(1 \mathrm{H}, \mathrm{b}, \mathrm{NH}), 8.02(1 \mathrm{H}, \mathrm{s}, \mathrm{CH}-5), 10.60 \mathrm{ppm}(1 \mathrm{H}, \mathrm{b}, \mathrm{NH}) .{ }^{13} \mathrm{C}-\mathrm{NMR}\left(\mathrm{DMSO}-\mathrm{d}_{6}\right): \delta$ 23.86 (C6'), 25.51 (C5'), 43.62 (C1'), 53.98 (C4'), 58.50 (C2'), 130.8 (C2), 133.8 (C3), 143.35 (C5), $149.70(\mathrm{C} 8), 161.92$ and $162.16 \mathrm{ppm}$ (C6 and $\mathrm{C} 10)$.

6-Imino-1-(2-pyrrolidin-1-yl-ethyl)-6,7-dihydro-1H,5H-1,3,5,7-tetraaza-azulene-4,8-dione (11): (55 mg; 13.99\%; m.p. $\left.>300{ }^{\circ} \mathrm{C}\right)$; MS m/z: $292.1(\mathrm{M}+1) .{ }^{1} \mathrm{H}-\mathrm{NMR}\left(\mathrm{DMSO}-d_{6}\right): \delta 1.90\left(4 \mathrm{H}, \mathrm{m}, \mathrm{CH}_{2}-5^{\prime}\right), 3.24$ $\left(4 \mathrm{H}, \mathrm{m}, \mathrm{CH}_{2}-4^{\prime}\right), 3.54\left(2 \mathrm{H}, \mathrm{m}, \mathrm{CH}_{2}-2^{\prime}\right), 4.68\left(2 \mathrm{H}, \mathrm{t}, J=6.1, \mathrm{CH}_{2}-1^{\prime}\right), 6.57(1 \mathrm{H}, \mathrm{b}, \mathrm{NH}), 7.68(1 \mathrm{H}, \mathrm{b}, \mathrm{NH})$, $8.19(1 \mathrm{H}, \mathrm{s}, \mathrm{CH}-5), 10.74 \mathrm{ppm}(1 \mathrm{H}, \mathrm{b}, \mathrm{NH}) .{ }^{13} \mathrm{C}-\mathrm{NMR}$ (DMSO-d $\left.\mathrm{D}_{6}\right): \delta 22.65$ (C5'), 43.06 (C1'), 53.79 (C4'), 54.14 (C2'), 130.7 (C2), 134.4 (C3), 143.5 (C5), 150.12 (C8), 159.15 and 161.97 ppm (C6 and C10). 


\subsubsection{General Procedure for Synthesis of $\mathbf{1 2 - 1 3}$}

To a solution of guanidine hydrochloride $(514 \mathrm{mg} ; 5.38 \mathrm{mmol})$ in anhydrous methanol $(8 \mathrm{~mL})$ cooled to $0{ }^{\circ} \mathrm{C}$ is added a solution of sodium methoxide $(25 \mathrm{wt} \% ; 0.75 \mathrm{~mL} ; 13.12 \mathrm{mmol})$. The reaction mixture is stirred for $30 \mathrm{~min}$ at $0{ }^{\circ} \mathrm{C}$. The sodium salt formed is removed by filtration, and the filtrate thus obtained is added to a solution of compound 1 or 2 (400 mg; $1.35 \mathrm{mmol})$ in anhydrous methanol $(5 \mathrm{~mL})$. Reaction mixture is stirred for $8 \mathrm{~h}$ at room temperature, solvent evaporated and in reaction mixture in situ dissolved in DMF (3 mL) is added 2-fluorophenyl isocyanate $(0,02 \mathrm{~mL} ; 0,18 \mathrm{mmol})$. Reaction mixture is stirred for $24 \mathrm{~h}$ at room temperature. The solvent is evaporated and the crude product purified by silica gel column chromatography $\left(\mathrm{CH}_{2} \mathrm{Cl}_{2} / \mathrm{CH}_{3} \mathrm{OH}=60 / 1\right)$ to give:

5-(\{[3-(2-Fluoro-phenyl)-ureido]-imino-methyl\}-carbamoyl)-1-(2-morpholin-4-yl-ethyl)-1H-imidazole4-carboxylic acid methyl ester (12) as a white crystals (6.5 mg; 1.63\%; m.p. > 164-165 $\left.{ }^{\circ} \mathrm{C}\right) ; \mathrm{MS} \mathrm{m} / z$ $462.1915(\mathrm{M}+1) .{ }^{1} \mathrm{H}-\mathrm{NMR}\left(\mathrm{DMSO}-d_{6}\right): \delta 2.48\left(4 \mathrm{H}, \mathrm{t}, J=4.7, \mathrm{CH}_{2}-4{ }^{\prime}\right), 2.73\left(2 \mathrm{H}, \mathrm{t}, J=6.0, \mathrm{CH}_{2}-2^{\prime}\right)$, $3.66\left(4 \mathrm{H}, \mathrm{t}, J=4.7, \mathrm{CH}_{2}-5^{\prime}\right), 4.10\left(3 \mathrm{H}, \mathrm{s}, \mathrm{COOCH}_{3}-3\right), 4.55\left(2 \mathrm{H}, \mathrm{t}, J=6.0, \mathrm{CH}_{2}-1^{\prime}\right), 6.97(1 \mathrm{H}, \mathrm{m}, \mathrm{F}-$ $\left.\mathrm{C}_{6} \mathrm{H}_{4}\right), 7.06\left(1 \mathrm{H}, \mathrm{m}, \mathrm{F}-\mathrm{C}_{6} \mathrm{H}_{4}\right), 7.11\left(1 \mathrm{H}, \mathrm{m}, \mathrm{F}-\mathrm{C}_{6} \mathrm{H}_{4}\right), 7.37(1 \mathrm{H}, \mathrm{b}, \mathrm{NH}), 7.71(1 \mathrm{H}, \mathrm{s}, \mathrm{CH}-5), 8.29(1 \mathrm{H}$, m, F-C $\left.\mathrm{C}_{6} \mathrm{H}_{4}\right), 8.83(1 \mathrm{H}, \mathrm{b}, \mathrm{NH}), 9.57(1 \mathrm{H}, \mathrm{b}, \mathrm{NH}), 12.48 \mathrm{ppm}(1 \mathrm{H}, \mathrm{b}, \mathrm{NH}) .{ }^{13} \mathrm{C}-\mathrm{NMR}$ (DMSO-d $\left.d_{6}\right): \delta$ 24.11 (C6'), 25.71 (C5'), 43.49 (C1'), 54.29 (C4'), 59.05 (C2'), $115.10\left(\mathrm{~F}_{-} \mathrm{C}_{6} \mathrm{H}_{4}, \mathrm{~d}, J_{\mathrm{CF}}=18.0\right), 116.5$ $\left(\mathrm{F}-\mathrm{C}_{6} \mathrm{H}_{4}\right), 121.43(\mathrm{C} 2), 124.80\left(\mathrm{~F}-\mathrm{C}_{6} \mathrm{H}_{4}, \mathrm{~d}, J_{\mathrm{CF}}=3.2\right), 136.3\left(\mathrm{~F}-\mathrm{C}_{6} \mathrm{H}_{4}\right), 139.97(\mathrm{C} 5), 145.53(\mathrm{C} 3)$, $150.95\left(\mathrm{~F}-\mathrm{C}_{6} \mathrm{H}_{4}, \mathrm{~d}, J_{\mathrm{CF}}=236.4\right), 158.20,161.73$ and $163.60(2 \times \mathrm{C}=\mathrm{O}$ and $\mathrm{C}=\mathrm{NH}), 167.55\left(\underline{\mathrm{COOCH}_{3}}\right)$. ${ }^{19} \mathrm{~F}$ NMR (DMSO- $\left.d_{6}\right): \delta-125.27\left(\mathrm{~F}-\mathrm{C}_{6} \mathrm{H}_{4}, \mathrm{~b}\right)$.

5-(\{[3-(2-Fluoro-phenyl)-ureido]-imino-methyl $\}$-carbamoyl)-3-(2-piperidin-1-yl-ethyl)-3H-imidazole4-carboxylic acid methyl ester (13) as a yellow oil (7.2 mg; 0.72\%); MS m/z 460.2 (M+1). ${ }^{1} \mathrm{H}-\mathrm{NMR}$ $\left(\mathrm{CDCl}_{3}\right): \delta 1.34\left(2 \mathrm{H}, \mathrm{m}, \mathrm{CH}_{2}-6^{\prime}\right), 1.42\left(4 \mathrm{H}, \mathrm{m}, \mathrm{CH}_{2}-5^{\prime}\right), 2.30\left(4 \mathrm{H}, \mathrm{m}, \mathrm{CH}_{2}-4^{\prime}\right), 2.5\left(2 \mathrm{H}, \mathrm{m}, \mathrm{CH}_{2}-2^{\prime}\right)$, $3.73\left(3 \mathrm{H}, \mathrm{s}, \mathrm{COOCH}_{3}-2\right), 4.17\left(2 \mathrm{H}, \mathrm{t}, J=6.2, \mathrm{CH}_{2}-1^{\prime}\right), 6.5-7.0\left(4 \times 1 \mathrm{H}, \mathrm{m}, \mathrm{F}-\mathrm{C}_{6} \mathrm{H}_{4}\right), 7.69(1 \mathrm{H}, \mathrm{s}$, $\mathrm{CH}-5), 7.7-8.6,12.3 \mathrm{ppm}(4 \times 1 \mathrm{H}, \mathrm{b}, \mathrm{NH}) .{ }^{13} \mathrm{C}-\mathrm{NMR}\left(\mathrm{CDCl}_{3}\right): \delta 46.15\left(\mathrm{C} 1^{\prime}\right), 53.79(\mathrm{C} 4), 53.86$ $\left(\mathrm{COOCH}_{3}\right), 58.60\left(\mathrm{C}^{\prime}\right), 66.90\left(\mathrm{C}^{\prime}\right), 114.70\left(\mathrm{~F}-\mathrm{C}_{6} \mathrm{H}_{4}, \mathrm{~d}, J_{\mathrm{CF}}=19.2\right), 120.47\left(\mathrm{~F}-\mathrm{C}_{6} \mathrm{H}_{4}\right), 122.66\left(\mathrm{~F}-\mathrm{C}_{6} \mathrm{H}_{4}\right.$, $\left.\mathrm{d}, J_{\mathrm{CF}}=7.1\right), 124.36\left(\mathrm{~F}-\mathrm{C}_{6} \mathrm{H}_{4}, \mathrm{~d}, J_{\mathrm{CF}}=3.3\right), 127.69\left(\mathrm{~F}-\mathrm{C}_{6} \mathrm{H}_{4}, \mathrm{~d}, J_{\mathrm{CF}}=16.5\right), 127.71(\mathrm{C} 2), 134.19(\mathrm{C} 3)$, $142.33(\mathrm{C} 5), 152.25\left(\mathrm{~F}-\mathrm{C}_{6} \mathrm{H}_{4}, \mathrm{~d}, J_{\mathrm{CF}}=242.8\right), 158.04,160.43$ and $162.98(2 \times \mathrm{C}=\mathrm{O}$ and $\mathrm{C}=\mathrm{NH}), 165.70$ $\left(\underline{\left.\mathrm{COOCH}_{3}\right) .}{ }^{19} \mathrm{~F} \mathrm{NMR}\left(\mathrm{CDCl}_{3}\right): \delta-124.37\left(\mathrm{~F}-\mathrm{C}_{6} \mathrm{H}_{4}, \mathrm{~b}\right)\right.$.

\subsection{Biological Methods}

\subsubsection{Cell Culturing}

The cell lines HeLa (cervical carcinoma), SW620 (colorectal adenocarcinoma, metastatic), MCF-7 (breast epithelial adenocarcinoma, metastatic), HepG2 (hepatocellular carcinoma) and BJ (normal diploid human fibroblasts), were cultured as monolayers and maintained in Dulbecco's modified Eagle medium (DMEM) supplemented with 10\% fetal bovine serum (FBS), $2 \mathrm{mM}$ L-glutamine, $100 \mathrm{U} / \mathrm{mL}$ penicillin and $100 \mu \mathrm{g} / \mathrm{mL}$ streptomycin in a humidified atmosphere with $5 \% \mathrm{CO}_{2}$ at $37^{\circ} \mathrm{C}$. 


\subsubsection{Proliferation Assays}

The panel cell lines were inoculated onto a series of standard 96-well microtiter plates on day 0 , at 3,000 to 5,000 cells per well according to the doubling times of specific cell line. Test agents were then added in 10-fold dilutions $(0,01$ to $100 \mu \mathrm{M})$ and incubated for further $72 \mathrm{~h}$. Working dilutions were freshly prepared on the day of testing in the growth medium. The solvent (DMSO) was also tested for eventual inhibitory activity by adjusting its concentration to be the same as in the working concentrations (DMSO concentration never exceeded $0.1 \%$ ). After $72 \mathrm{~h}$ of incubation, the cell growth rate was evaluated by performing the MTT assay: experimentally determined absorbance values were transformed into a cell percentage growth (PG) using the formulas proposed by NIH and described previously [10]. This method directly relies on control of untreated cells at the day of substances addition because it compares the growth of treated cells with the growth of untreated cells in control wells on the same plate - the results are therefore a percentile difference from the calculated expected value. The $\mathrm{IC}_{50}$ values for each compound were calculated from dose-response curves using linear regression analysis by fitting the mean test concentrations that give PG values above and below the reference value. If, however, all of the tested concentrations produce PGs exceeding the respective reference level of effect (e.g., PG value of 50) for a given cell line, the highest tested concentration is assigned as the default value (in the screening data report that default value is preceded by a ">" sign). Each test point was performed in quadruplicate in three individual experiments. The results were statistically analyzed (ANOVA, Tukey post-hoc test at $p<0.05$ ). Finally, the effects of the tested substances were evaluated by plotting the mean percentage growth for each cell type in comparison to control on dose response graphs.

\subsubsection{Antiviral Activity Assays}

The antiviral assays, other than the anti-HIV assays, were based on inhibition of virus-induced cytopathicity in HEL [herpes simplex virus type 1 (HSV-1) (KOS), HSV-2 (G), vaccinia virus and vesicular stomatitis virus], Vero (parainfluenza-3, reovirus-1, Sindbis, Coxsackie B4, and Punta Toro virus), HeLa (vesicular stomatitis virus, Coxsackie virus B4, and respiratory syncytial virus) or MDCK [influenza A (H1N1; H3N2) and influenza B] cell cultures. Confluent cell cultures (or nearly confluent for MDCK cells) in microtiter 96-well plates were inoculated with $100 \mathrm{CCID}_{50}$ of virus $\left(1 \mathrm{CCID}_{50}\right.$ being the virus dose to infect $50 \%$ of the cell cultures). The cell cultures were incubated at the time of infection in the presence of varying concentrations $(200,40,8, \ldots \mu \mathrm{M})$ of the test compounds. Viral cytopathicity was recorded microscopically as soon as it reached completion in the control virus-infected cell cultures that were not treated with the test compounds. The methodology of the anti-HIV assays was as follows: human CEM cells $\left(\sim 3 \times 10^{5}\right.$ cells $\left./ \mathrm{mL}\right)$ were infected with $100 \mathrm{CCID}_{50}$ of $\mathrm{HIV}\left(\mathrm{III}_{\mathrm{B}}\right)$ or $\mathrm{HIV}-2(\mathrm{ROD}) / \mathrm{mL}$ and seeded in $200-\mu \mathrm{L}$ wells of a microtiter plate containing appropriate dilutions of the test compounds. After 4 days of incubation at $37{ }^{\circ} \mathrm{C}$, HIV-induced giant cell formation was examined microscopically. 


\section{Conclusions}

The main objective of this study was synthesis of a molecule that would be closely related to RSV604. None of the compounds showed pronounced antiviral activity at subtoxic concentrations. No cytotoxicity for all evaluated compounds on HEL, Vero, HeLa and MDCK cell cultures was observed.

From the structure-activity point of view, we believe that the main reason for lack of antiviral activity is probably the absence of either sugar moiety at $N-1$ of imidazole or a closer resemblance to the active compound RSV604, that was chemically caused and constrained by the formation of the inactive imino form of the cyclized product instead of the targeted amino form. The $1 H$-imidazole-4,5dicarboxylic acid dimethyl ester derivative having an ethyl morpholino ligand (compound 1) and the $1 H$-imidazole-4,5-diamide derivative with morpholino moiety bound to an imidazole ring (compound 5) have also exerted selective but modest cytostatic effect towards human cervix carcinoma (HeLa) cells $\left(\mathrm{IC}_{50}=24\right.$ and $\left.32 \mu \mathrm{M}\right)$, while moderate non-selective antiproliferative activity was observed for compound 10. The imidazole[4,5-e][1,3]diazepine-4,8-dione derivative linked with a pyrrolidine ligand (11) showed only a modest cytostatic effect on colon cancer cells (SW620) $\left(\mathrm{IC}_{50}=20 \mu \mathrm{M}\right)$. These compounds showed no cytotoxic effects on human normal fibroblasts.

Synthetic chemistry is often full of surprises and presented compounds containing a carbamoyl imino-ureido moiety are structurally very interesting, e.g., compounds $\mathbf{1 2}$ and $\mathbf{1 3}$. Moreover, $1 \mathrm{H}$-imidazole derivative containing a pyrrolidine moiety linked via an ethylenic spacer to $\mathrm{N}$ - 1 of the imidazole ring (compound 3) exerted a more pronounced selective cytostatic effect towards human cervix cancer $(\mathrm{HeLa})$ cells $\left(\mathrm{IC}_{50}=9.5 \mu \mathrm{M}\right)$ in comparison with other tested compounds with no apparent cytotoxicity on normal human skin fibroblasts (BJ) as well. This compound might therefore be suited for further exploration as a potential lead compound and chemical modifications.

\section{Acknowledgments}

Support for this study was provided by the Ministry of Science of the Republic of Croatia (Projects \#125-098-2464-2922, \#335-0982464-2393, \#335-0000000-3532). The research was supported by the KU Leuven (GOA no. 10/014).

\section{Conflicts of Interest}

The authors declare no conflict of interest.

\section{References}

1. Collins, P.L.; Chancock, R.M.; Murphy, B.R. Respiratory Syncytial Virus. In Fields Virology, 4th ed.; Knipe, D.M., Howley, P.M., Eds.; Lippincott Williams \& Wilkins: Philadelphia, PA, USA, 2001; Volume 1, pp. 1443-1485.

2. Anderson, L.J.; Parker, R.A.; Strikas, R.L. Association between respiratory syncytial virus outbreaks and lower respiratory tract deaths of infants and young children. J. Infect. Dis. 1990, 161, 640-646.

3. Meanwell, N.A.; Krystal, M. Respiratory syncytial virus: Recent progress toward the discovery of effective profylactic and therapeutic agents. Drug Discov. Today 2000, 5, 241-252. 
4. Carter, M.C.; Alber, D.G.; Baxter, R.C.; Bithell, S.K.; Budworth, J.; Chubb, A.; Cockerill, G.S.; Dowdell, V.C.L.; Henderson, E.A.; Keegan, S.J.; et al. 1,4-Benzodiazepines as inhibitors of respiratory syncytial virus. J. Med. Chem. 2006, 49, 2311-2319.

5. Henderson, E.A.; Alber, D.G.; Baxter, R.C.; Bithell, S.K.; Budworth, J.; Carter, M.C.; Chubb, A.; Cockerill, G.S.; Dowdell, V.C.; Fraser, I.J.; et al. 1,4-Benzodiazepines as inhibitors of respiratory syncytial virus. The identification of a clinical candidate. J. Med. Chem. 2007, 50, 1685-1692.

6. Bhatnagar, A.; Sharma, P.K.; Kumar, N.; Dudhe, R. "Imidazoles" their chemistry and pharmacological potential. Int. J. PharmTech Res. 2011, 3, 268-282.

7. Xie, M.; Ujjinamatada, R.K.; Sadowska, M.; Lapidus, R.G.; Edelman, M.J.; Hosmane, R.S. A novel, broad spectrum anti-cancer compound containing the imidazo[4,5-e][1,3]diazepine ring system. Bioorg. Med. Chem. Lett. 2010, 20, 4386-4389.

8. Zhang, P.; Zhang, N.; Korba, B.E.; Hosmane, R.S. Synthesis and in vitro anti-hepatitis B and $\mathrm{C}$ virus activities of ring-expanded ('fat') nucleobase analogues containing the imidazo[4,5-e][1,3]diazepine-4,8-dione ring system. Bioorg. Med. Chem. Lett. 2005, 15, 5397-5401.

9. Zhang, N.; Chen, H.-M.; Sood, R.; Kalicharran, K.; Fattom, A.I.; Naso, R.B.; Barnard, D.L.; Sidwell, R.W.; Hosmane, R.S. In vitro inhibition of measles virus replication by novel ring-expanded ("fat") nucleoside analogues containing the imidazo[4,5-e][1,3]diazepine ring system. Bioorg. Med. Chem. Lett. 2002, 12, 3391-3394.

10. Gazivoda, T.; Raić-Malić, S.; Krištafor, V.; Makuc, D.; Plavec, J.; Bratulić, S.; Kraljević-Pavelić, S.; Pavelić, K.; Naesens, L.; Andrei, G.; et al. Synthesis, cytostatic and anti-HIV evaluations of the new unsaturated acyclic C-5 pyrimidine nucleoside analogues. Bioorg. Med. Chem. 2008, 16, $5624-5634$.

Sample Availability: Samples of the compounds 1-11 are available from the authors.

(C) 2013 by the authors; licensee MDPI, Basel, Switzerland. This article is an open access article distributed under the terms and conditions of the Creative Commons Attribution license (http://creativecommons.org/licenses/by/3.0/). 